Pacific Journal of Mathematics

ALGEBRAS OF ANALYTIC FUNCTIONS IN THE PLANE 


\title{
ALGEBRAS OF ANALYTIC FUNCTIONS IN THE PLANE
}

\author{
WILLIAM R. ZAME
}

Let $X$ be a compact subset of the complex plane and let $A$ be an algebra of functions analytic near $X$ which contains the polynomials and is complete in its natural topology. This paper is concerned with determining the spectrum of $A$ and describing $A$ in terms of its spectrum. It is shown that the spectrum of $A$ is formed from the disjoint union of certain compact subsets of $C$ (suitably topologized) by making certain identifications. $A$ is closed under differentiation exactly when no identifications need be performed, and then $A$ admits a simple, complete description. In particular, if $X$ is connected, then the completion of $A$ is merely the restriction to $X$ of the algebra of all functions analytic near the union of $X$ with some of the bounded components of $C-X$.

Our principal tool in these investigations is the theory of analytic structure in the spectrum of a function algebra developed by Bishop in [2] and extended by Bjork in [4,5]. We view the algebra $A$ as the inductive limit of function algebras and induce analytic structure in the spectrum of $A$. When $A$ is closed under differentiation, topological considerations lead quickly to the desired results. In the general case, we pass to the smallest algebra $B$ containing $A$ which is closed under differentiation. By introducing differentiation in the spectrum of $A$, we show that every continuous complex-valued homomorphism of $A$ may be extended to $B$. It follows that the spectrum of $A$ is obtained from the spectrum of $B$ by making certain identifications. When no identifications need be performed, $A=B$.

2. Preliminaries. If $U$ is an open set, we let $O(U)$ denote the algebra of functions analytic on $U$, endowed with the topology of uniform convergence on compact sets. If $V$ is an open subset of $U$, we let $r_{U V}: \mathscr{O}(U) \rightarrow \mathscr{O}(V)$ be the restriction. If $X$ is a compact set, $\mathcal{O}(X)$ denotes the algebra of functions on $X$ which have analytic extensions to a neighborhood of $X$. We view $\mathscr{O}(X)$ as the inductive limit (in the sense of functions) of the system $\left\{\mathcal{O}(U) ; r_{U V}\right\}$ and equip $\mathscr{O}(X)$ with the inductive limit topology; i.e., the finest topology rendering the restriction maps $r_{U}: \mathscr{O}(U) \rightarrow \mathscr{O}(X)$ continuous.

If $A$ is a subalgebra of $\mathscr{O}(X)$ and $U$ is an open set containing $X$, we let $A(U)=\{f \in \mathcal{O}(X): f \mid X \in A\}$. Similarly, if $K$ is a compact set containing $X$, we let $A(K)=\{f \in \mathcal{O}(K): f \mid X \in A\}$. For compact sets $K, L$ with $K \supset L$, we let $r_{K L}: O^{\circ} \rightarrow \mathcal{O}(L)$ be the restriction. Then it is easy to see that: 


$$
\begin{aligned}
A & =\text { inductive limit }\left\{A(U) ; r_{U V}\right\} \\
& =\text { inductive limit }\left\{A(K) ; r_{K L}\right\}
\end{aligned}
$$

and that the inductive limit topologies thus induced on $A$ coincide with the relative topology from $\mathscr{O}(X) . A$ is complete in this topology if and only if $A(U)$ is closed in $\mathscr{O}(U)$ for each open set $U$ containing $X$. Details of the above may be found in $\S 2$ of [11].

We also regard a subalgebra $A$ of $O(X)$ as a normed algebra with the norm:

$$
\|f\|_{X}=\sup \{|f(x)|: x \in X\} .
$$

We relate these two topologies in the following proposition.

Proposition. Let $A$ be a subalgebra of $\mathscr{O}(X)$ containing the constants. Then the norm topology and the inductive limit topology on $A$ admit the same continuous complex-valued homomorphisms.

Proof. If this were not so, there would be a homomorphism $\phi$ of $A$, continuous relative to the inductive limit topology, and a function $f$ in $A$ such that

$$
\phi(f)=1>\|f\|_{X} \cdot
$$

We could then find an open set $U$ containing $X$ and a function $F$ in $A(U)$ such that $F \mid X=f$ and $|F|<1$ on $U$. Then $(1-F)$ would be invertible in the closure of $A(U)$ in $\mathcal{O}(U)$. Moreover, $\phi \cdot r_{U}$ would be a continuous homomorphism of $A(U)$, and would thus extend to its closure. Since $\phi \cdot r_{U}(1-F)=\phi(1-f)=0$, we would then have the following contradictory chain of equalities:

$$
1=\phi \cdot r_{U}\left[(1-F)(1-F)^{-1}\right]=\left\{\phi \cdot r_{U}(1-F)\right\}\left\{\phi \cdot r_{U}\left[(1-F)^{-1}\right]\right\}=0 .
$$

This contradiction establishes the proposition.

If $B$ is a topological algebra, we denote the spectrum of $B$ (the space of nonzero continuous complex-valued homomorphisms, with the weak* topology) by $M_{B}$. We may regard an element $b$ of $B$ as a function of $M_{B}$ via the Gelfand transform $\hat{b}(\phi)=\phi(b)$, for each $\phi$ in $M_{B}$. If $B$ is a normed algebra with identity, then $M_{B}$ is compact. Then if $A$ is a subalgebra of $\mathcal{O}(X)$ containing the constants, $M_{A}$ is compact and a standard argument may be used to show that (see [11]):

$$
\begin{aligned}
M_{A} & =\text { projective limit }\left\{M_{A(U)} ; r_{U V}^{*}\right\} \\
& =\text { projective limit }\left\{M_{A(K)} ; r_{K L}^{*}\right\}
\end{aligned}
$$

where $r_{U V}^{*}$ and $r_{K L}^{*}$ are the adjoints of the restrictions. 
We refer to [6] for standard material concerning function algebras. If $B$ is a function algebra with spectrum $M_{B}$ we denote its Silov boundary by $S_{B}$. We make use of the techniques developed by Bishop and Bjork in $[2,4,5]$ and assume some familiarity with these papers. In particular, if $f$ is an element of $B$ we say that a component $W$ of $C-\hat{f}\left(S_{B}\right)$ is $f$-regular of multiplicity $n$ if for each $w$ in $W$ there are at most $n$ homomorphisms $\zeta$ in $M_{B}$ for which $\hat{f}(\zeta)=w$; and that for some $w$ there are exactly $n$ such homomorphisms. In that case, there is a discrete subset $E$ of $W$ such that for each $\zeta$ in $M_{B}$ such that $\hat{f}(\zeta) \in(W-E)$, there is a neighborhood $Q$ of $\zeta$ in $M_{B}$ mapped homeomorphically by $\hat{f}$ onto a disk, and such that $\hat{g} \circ(\hat{f} \mid Q)^{-1}$ is analytic for each $g$ in $B$. The neighborhood $Q$ is called an analytic disk about $\zeta$, relative to the function $\hat{f}$.

We conclude this section with a topological lemma.

Lemma. Let $M$ be a compact connected real 2-manifold with boundary and let $p$ be a continuous map of $M$ into the 2-sphere $S^{2}$. If $p$ is locally one-to-one and is one-to-one on the boundary of $M$, then $p$ is one-to-one.

Proof. We will reduce to the case of a 2-manifold without boundary. To this end, suppose that $M$ has $k$ boundary components $J_{1}, \cdots, J_{k c}$. Each $J_{i}$ is a 1 -sphere, so that $p\left(J_{i}\right)$ is a 1 -sphere in $S^{2}$ for each $i$. Hence $S^{2}-p\left(J_{i}\right)$ consists of two disjoint connected open sets. A compactness argument, using the fact that $p$ is locally-oneto-one, may be used to show that there is a connected neighborhood of $J_{i}$ in $M$ on which $p$ is one-to-one. It follows that we may choose a neighborhood $W_{i}$ of $J_{i}$ such that $p$ is one-to-one on $W_{i}$ and $p\left(W_{i}\right)$ does not intersect one of the components of $S^{2}-p\left(J_{i}\right)$. It is easy to see that we may attach a disk to $M$ along $J_{i}$ and extend $p$ to this disk; since $p\left(W_{i}\right)$ lies in only one component of $S^{2}-p\left(J_{i}\right)$ this may be effected in such a way that the extension remains locally one-toone. If we perform this surgery for each boundary component $J_{i}$ we arrive at a compact connected real 2-manifold $N$ without boundary and a continuous map $q$ of $N$ into $S^{2}$ which is locally one-to-one. If $q$ is one-to-one then $p$ must certainly be.

For each $x$ in $S^{2}$, the fiber $q^{-1}(x)$ is compact and discrete (since $q$ is locally one-to-one) and hence finite. Then, using the invariance of domain, we may choose an open set $U$ about $x$ such that $q^{-1}(U)$ consists of open, connected components, each mapped homeomorphically onto $U$ by $q$; thus $q$ is a covering map. Since $S^{2}$ is its own universal covering space, it follows that $q$, and hence $p$, must be one-to-one, as desired. 
3. Main results. If $A$ is a subalgebra of $\mathscr{O}(X)$ that contains the constants and the coordinate function $Z$, we say that $A$ is stable if it is complete in the inductive limit topology and each of the algebras $A(U)$ is closed under differentiation.

In order to see how stable algebras may arise, consider the following construction. Let $X$ be a compact subset of $C$ and let $\left\{X_{\alpha}\right\}$ be a partitioning of $X$ into disjoint closed sets. For each $\alpha$ let $Y_{\alpha}$ be the union of $X_{\alpha}$ with some of the bounded components of $C-X_{\alpha}$. Then let

$$
A=\left\{f \in \mathcal{O}(X): f\left|X_{\alpha} \in \mathcal{O}\left(Y_{\alpha}\right)\right| X_{\alpha} \text { for each } \alpha\right\} .
$$

It is easy to see that $A$ is a stable algebra and that the spectrum of $A$ is the disjoint union of the $Y_{\alpha}$, suitably topologized. The following theorem shows that this is the only way in which stable algebras may arise.

Theorem 1. Let $A$ be a stable subalgebra of $\mathscr{O}(X)$ and let $Y_{\alpha}^{\prime}$ be a component of $M_{A}$. Then $\hat{Z} \mid Y_{\alpha}^{\prime}$ is a homeomorphism. The set $Y_{\alpha}=$ $\hat{Z}\left(Y_{\alpha}^{\prime}\right)$ is the union of $X_{\alpha}=X \cap Y_{\alpha}$ with some of the bounded components of $C-X_{\alpha}$. Finally, the collection $\left\{X_{\alpha}: Y_{\alpha}^{\prime}\right.$ is a component of $\left.M_{A}\right\}$ is a partitioning of $X$ into disjoint closed sets and $A=\{f \in \mathcal{O}(X)$ : $f\left|X_{\alpha} \in \mathcal{O}\left(Y_{\alpha}\right)\right| X_{\alpha}$ for each component $Y_{\alpha}^{\prime}$ of $\left.M_{A}\right\}$.

Proof. Let $K$ be a compact set whose interior contains $X$ and whose boundary is the disjoint union of a finite number of smooth, simple closed curves. Let $A(K)^{*}$ denote the completion of the algebra $A(K)$ in the norm $\|\cdot\|_{K}$. We proceed by examining the algebra $A(K)^{*}$ and its spectrum and then passing to the projective limit.

We identify $K$ with a subset of $M_{A(K) *}$. Clearly, $S_{A(K) *}$ is contained in the boundary (relative to $C$ ) of $K$. Let $\Lambda$ denote the set of points in $M_{A(K)^{*}}$ having a neighborhood which is an analytic disk (relative to the function $\hat{Z})$. We show that $M_{A(K)^{*}}-S_{A(K)^{*}}-\Lambda$ is at most countable. First, a standard argument shows that the unbounded component of $C-\hat{Z}\left(S_{\left.A(K)^{*}\right)}\right.$ is $Z$-regular of multiplicity 0 . If $T$ is the boundary of this component, then it follows from [5] that there are no points $\zeta$ of $M_{A(K)^{*}}-S_{A(K)^{*}}$ for which $\hat{Z}(\zeta)$ belongs to $T$. We conclude from [5] that each component of $C-\hat{Z}\left(S_{A(K) *}\right)$ that adjoins the unbounded component is $Z$-regular of multiplicity at most 1 . Similarly, if $T^{\prime}$ denotes the boundary of one of these components, then there is at most one point $\zeta$ in $M_{A(K)^{*}}-S_{A(K)^{*}}$ for which $\hat{Z}(\xi) \in T^{\prime}$. Then each component of $C-\hat{Z}\left(S_{A(K) *}\right)$ that adjoins one of these components is $Z$-regular of multiplicity at most 2. Proceeding inward in this way, we see that each component of $C-\hat{Z}\left(S_{A(K)^{*}}\right)$ is $Z$-regular of some multiplicity. Again from [5], it follows that there is a discrete subset 
$E$ of $C-\hat{Z}\left(S_{A(K) *}\right)$ such that each $\xi$ in $M_{A(K)^{*}}-S_{A(K)^{*}}$ for which $\hat{Z}(\xi)$ does not lie in $E \cup \hat{Z}\left(S_{\left.A(K)^{*}\right)}\right.$, is a point of $\Lambda$. Moreover, if $x$ is in $E$, then there are only finitely many homomorphisms $\psi$ for which $\hat{Z}(\psi)=x$.

Now let us turn to the points $\xi$ of $M_{A(K)^{*}}-S_{A(K)^{*}}$ for which $\hat{Z}(\xi) \in$ $\hat{Z}\left(S_{A(K)^{*}}\right)$. Since the boundary of $K$ is the finite union of smooth curves, it follows that each boundary point is a triangle point in the sense of Bishop [2]. Hence for each $\xi$ in $M_{A(K)^{*}}-S_{A(K)^{*}}$ for which $\hat{Z}(\xi) \in$ $\hat{Z}\left(S_{A(K)^{*}}\right)$, there is a deleted neighborhood $W_{\xi}$ lying in $\Lambda$. From the compactness of the boundary of $K$ and the fact that for each point $y$ of the boundary there are only finitely many homomorphisms $\zeta$ for which $\hat{Z}(\zeta)=y$, it follows that all but finitely many of the points $\xi$ of $M_{A(K)^{*}}-S_{A(K)^{*}}$ for which $\hat{Z}(\hat{\xi}) \in \hat{Z}\left(S_{A(K)^{*}}\right)$ actually lie in $\Lambda$. Then $M_{A(K)^{*}}-S_{A(K)^{*}}-\Lambda$ is a countable set, as was asserted.

Now let $L$ be a connected component of $M_{A(K) *}$. We assert that $\hat{Z} \mid L$ is a homeomorphism. If this were not so, we could find homomorphisms $\phi$ and $\lambda$ in $L$ such that $\hat{Z}(\phi)=\hat{Z}(\lambda)$. Since $M_{A(K)^{*}}=M_{A(K)}$, we could then find an open set $U$ containing $K$ and a function $f$ in $A(U)$ such that $\phi(f \mid K) \neq \lambda(f \mid K)$. Since $A(U)$ is complete, closed under differentiation and contains the polynomials, it follows from a theorem of Bishop [3] that $M_{A(C)}$ is a 1-dimensional complex analytic manifold and that $\hat{Z}$ on $M_{A\left(C^{\circ}\right)}$ is a local analytic isomorphism. Let $\rho: A(U) \rightarrow A(K)$ be the restriction and $\rho^{*}: M_{A(K)} \rightarrow M_{A(U)}$ be its adjoint. Then $\rho^{*}(L)$ is a compact connected subset of $M_{A(U)}$. By the invariance of domain theorem, $\rho^{*}(\Lambda)$ lies in the interior of $\rho^{*}(L)$. Hence $\hat{Z}$ is one-to-one on the boundary of $\rho^{*}(L)$. Since $\hat{Z}$ is a local homeomorphism on $M_{A(U)}$, we may find a compact connected set $L^{\prime}$ containing $\rho^{*}(L)$ in its interior such that $\hat{Z}$ is one-to-one on the boundary of $L^{\prime}$ and $L^{\prime}$ is a 2 -manifold with boundary. Regarding $C$ as a subset of $S^{2}$, we may then apply the lemma to conclude that $\hat{Z}$ is one-to-one on $L^{\prime}$ and hence on $\rho^{*}(L)$. But $\phi$ and $\lambda$ restrict to different homomorphisms of $A(U)$ so that $\rho^{*}(\dot{\phi}) \neq \rho^{*}(\lambda)$, while $\hat{Z}\left[\rho^{*}(\phi)\right]=\hat{Z}\left[\rho^{*}(\lambda)\right]$, which is a contradiction. It must be therefore, that $\hat{Z} \mid L$ is a homeomorphism.

From the Silov idempotent theorem, it follows that each component of $M_{A(K)^{*}}$ contains a component of the boundary of $K$. It follows that for each component $L$ of $M_{A(K)^{*}}$, the boundary of $\hat{Z}(L)$ coincides with $\hat{Z}\left(S_{A(K) *} \cap L\right)$, so that $\hat{Z}(L)$ is formed from $K \cap \hat{Z}(L)$ by the addition of certain components of $C-K \cap \hat{Z}(L)$.

Now let us return to $M_{.}$. For a component $Y_{\alpha}^{\prime}$ of $M_{A}$, and a compact set $K$ with smooth boundary, containing $X$ in its interior, let $r_{K}: A(K)^{*} \rightarrow A$ be the restriction and let $r_{K}^{*}: M_{A} \rightarrow M_{A(K) *}$ be its adjoint. Let $K_{\alpha}^{\prime}$ be the component of $M_{A(K)^{*}}$ that contains $r_{K}\left(Y_{\alpha}\right)$. It is clear that

$$
Y_{a}^{\prime}=\text { projective limit }\left\{K_{\alpha}^{\prime} ; r_{K L}^{*}\right\} \text {. }
$$


From the description of $K_{\alpha}^{\prime}$ derived above, it follows that $\hat{Z} \mid Y_{\alpha}^{\prime}$ is a homeomorphism and that $\hat{Z}\left(Y_{\alpha}^{\prime}\right)$ is the union of $X \cap \hat{Z}\left(Y_{\alpha}^{\prime}\right)=X_{\alpha}$ with some of the bounded components of $C-X_{\alpha}$.

If $f$ belongs to $A$, then it is in $A(K)$ * for some compact $K$ with smooth boundary containing $X$ in its interior. Since $\hat{Z}$ is a homeomorphism on each component of $M_{A\langle K\rangle}$, it follows that $A$, the set of points in $M_{A(K)^{*}}$ having neighborhoods which are analytic disks, is all of $M_{A^{(K)^{*}}}-S_{A(K)^{*}}$. Now we may see that $f \mid\left(K \cap \hat{Z}\left(L^{\prime}\right)\right)$ belongs to $\mathscr{O}\left(\hat{Z}\left(L^{\prime}\right)\right) \mid\left(K \cap \hat{Z}\left(L^{\prime}\right)\right)$ for each component $L^{\prime}$ of $M_{A(K) *}$. It follows that $f \mid\left(X \cap \hat{Z}\left(Y_{\alpha}^{\prime}\right)\right)$ belongs to $\mathscr{O}\left(\hat{Z}\left(Y_{\alpha}^{\prime}\right)\right) \mid\left(X \cap \hat{Z}\left(Y_{\alpha}^{\prime}\right)\right)$ for each component $Y_{\alpha}^{\prime}$ of $M_{A}$.

Finally, suppose that $U$ is an open set containing $X$ and that $f$ is a function in $\mathcal{O}(U)$ such that $f\left(\left(X \cap \hat{Z}_{(} Y_{\alpha}^{\prime}\right)\right)$ belongs to

\section{$\mathscr{O}\left(\hat{Z}\left(Y_{\alpha}^{\prime}\right)\right) \mid\left(X \cap \hat{Z}\left(Y_{a}^{\prime}\right)\right)$}

for each component $Y_{\alpha}^{\prime}$ of $M_{A}$. For each such $Y_{\alpha}^{\prime}$, choose a compact set $K_{\alpha}$ with smooth boundary containing $X$ in its interior and such that $Z\left(L_{\alpha}^{\prime}\right) \subset\left(U \cup \hat{Z}\left(Y_{\alpha}^{\prime}\right)\right)$ where $L_{\alpha}^{\prime}$ is the component of $M_{A(K)^{*}}$ that contains $r_{K}^{*}\left(Y_{\alpha}^{\prime}\right)$. If $Y_{\beta}^{\prime}$ is sufficiently close to $Y_{a}^{\prime}$, we may choose $K_{\beta}$ to be $K_{\alpha}$. Then the compactness of $M_{A}$ enables us to choose a single compact set $K^{\prime}$ with smooth boundary, containing $X$ in its interior, and such that $\hat{Z}\left(L_{\alpha}^{\prime \prime}\right) \subset\left(U \cup \hat{Z}\left(Y_{\alpha}^{\prime}\right)\right)$ for each $\alpha$, where $L_{\alpha}^{\prime \prime}$ is the component of $M_{A\left(K^{\prime}\right)}{ }^{*}$ that contains $r_{K^{\prime}}^{*}\left(Y_{a}^{\prime}\right)$. Without loss, we may assume that every component of $K^{\prime}$ contains a point of $X$. Then for each component $L^{\prime}$ of $M_{A\left(K^{\prime}\right) *}$ we see that $f \mid\left(K^{\prime} \cap \hat{Z}\left(L^{\prime}\right)\right)$ belongs to $\mathcal{O}\left(\hat{Z}\left(L^{\prime}\right)\right) \mid\left(K^{\prime} \cap \hat{Z}\left(L^{\prime}\right)\right)$. The Silov idempotent theorem and the Arens-Calderon theorem then imply that $f \mid K^{\prime}$ belongs to $A\left(K^{\prime}\right)^{*}$. Since $A$ is complete and $K^{\prime}$ contains $X$ in its interior, it follows that $f \mid X$ belongs to $A$, which completes the proof.

The above theorem gives a complete description of stable algebras. In what follows, we use stable algebras to describe the structure of more general subalgebras of $\mathscr{O}(X)$. We let $A$ be a complete subalgebra of $\mathcal{O}(X)$ containing the polynomials and let $A_{0}$ be the smallest stable algebra containing $A ; A_{0}$ is the completion of the algebra generated by the functions in $A$ together with all their derivatives. We let $i: A \rightarrow A_{0}$ be the inclusion and $i^{*}: M_{A_{0}} \rightarrow M_{A}$ be its adjoint (the restriction map).

Theorem 2. The map $i^{*}: M_{A_{0}} \rightarrow M_{A}$ is onto. If $Y$ and $Y^{\prime}$ are components of $M_{A_{0}}$ then $i^{*} \mid Y$ and $i^{*} \mid Y^{\prime}$ are one-to-one and there are at most finitely many pairs $(\mu, \nu)$ in $Y \times Y^{\prime}$ such that $i^{*}(\mu)=i^{*}(\nu)$. If $f^{*}$ is a homeomorphism, then $A=A_{0}$. 
Proof. We show first that $i^{*}$ is onto. Choose a compact set $K$ with smooth boundary, whose interior contains $X$ and is dense in $K$, and each component of which meets $X$. Let $A_{1}$ be the (non-complete) subalgebra of $\mathscr{O}(X)$ generated by the functions in $A$ and all their derivatives. Let $i_{K}: A(K) \rightarrow A_{1}(K)$ be the inclusion and $i_{K}^{*}: M_{A_{1}(K) *} \rightarrow$ $M_{A(K) *}$ be its adjoint. If we show that $i_{K}^{*}$ is onto for each $K$ belonging to a fundamental system of neighborhoods of $X$, then by passage to the projective limit, it will follow that $i^{*}$ is onto. So suppose that for a particular choice of $K, i_{K}^{*}$ is not onto.

Let $\Lambda$ be the set of points of $M_{A(K) *}$ which have a neighborhood which is an analytic disk relative to $\hat{Z}$. As in the proof of Theorem 1, we see that $M_{A(K)^{*}}-S_{A(K)^{*}}-\Lambda=E$ is at most countable. In view of the Silov idempotent theorem, no point of $M_{A(K) *}$ is isolated, so that there is an open subset of $\Lambda$ disjoint from $i_{K}^{*}\left(M_{A_{1}(K) *}\right)$. Let $W$ be a component of $\Lambda$ containing such an open set. We distinguish two cases.

Regard $K$ as a subset of $M_{A(K) *}$ and consider first the case in which $W$ contains a point of $K$. Then $W$ is a Riemann surface with the local coordinate $\hat{Z}$. If $f$ is a function in $A(K)$, then $\hat{f}$ is analytic on $W$. Denote the derivative of $\hat{f}$ with respect to the coordinate $\hat{Z}$ by $D \hat{f}$ and the derivative of $f$ with respect to $Z$ by $f^{\prime}$. If $f$ and $f^{\prime}$ both belong to $A(K)$, then the connectedness of $W$, together with the fact that $W$ contains a point of $K$ and hence an open subset of $K$, implies that $D \hat{f}=\hat{f}^{\prime}$ on $W$.

Let $h$ belong to $A(K)$ and let $g=h^{\prime}$. Define a function $\widetilde{g}$ on $W$ by $\widetilde{g}(\zeta)=D \hat{h}(\zeta)$. The analysis of the previous paragraph implies that $\widetilde{g}=\widehat{g}$ if $g=h^{\prime}$ belongs to $A(K)$. Thus the functions in $A(K)$ together with their first derivatives, extend to be analytic on $W$. By iteration of this process, we may extend each of the functions $g$ in $A_{1}(K)$ to an analytic function $\widetilde{g}$ on $W$; since $W$ is connected, this extension is unique.

Thus if $\delta$ is a homomorphism in $W, \delta$ extends to a homomorphism of $A_{1}(K)$ by defining $\bar{\delta}(g)=\widetilde{g}(\delta)$. If we show that $\bar{\delta}$ is a continuous homomorphism of $A_{1}(K)$, and thus extends to $A_{1}(K)^{*}$, then we will have that $i_{K}^{*}(\bar{\delta})=\delta$ and this contradiction will complete the analysis of this case.

To this end, let us consider the boundary of $W$ in $M_{A(K) *}$. Since $W$ is a connected component of $\Lambda$, no point of $\Lambda$ is a boundary point of $W$. Thus the boundary points of $W$ belong either to $K$ or to $E$. If $p$ is a boundary point of $W$ that belongs to $K$, the fact that the interior of $K$ is dense in $K$ and that the boundary of $K$ is smooth implies that there is a half-disk about $p$ belonging to $K$. By enlarging $K$ slightly we may effect a modification of $K$ so that some halfdisk around $p$ belongs to $K \cap W$. An argument using the compactness of the part of the boundary of $W$ that lies in $K$ shows that all the 
boundary points of $W$ that belong to $K$ may be assumed to have half-disks in $K \cap W$ about them (modifying $K$ as necessary).

Now consider the boundary points of $W$ that belong to $E$. If $q$ is one of these points, then the results of [2] imply that there is a neighborhood $Q$ of $q$ with the property that $Q-q$ lies in $A$ and consists of finitely many components, each of which is mapped by $\hat{Z}$ homeomorphically onto a disk minus its center. Thus we may cover $W \cup\{q\}$ with a Riemann surface $W_{q}$ in such a way that the functions in $A(K)$ extend to be analytic on $W_{q}$. We may certainly do this for each of the boundary points of $W$ lying in $E$. Thus, passing to a covering Riemann surface when necessary, and modifying $W$ as necessary (by enlargement of $K$ ), we arrive at a Riemann surface $W^{\prime}$ which has a subset of the interior of $K$ as a neighborhood of its boundary, and to which the functions in $A(K)$ extend naturally. As before, we see that the functions in $A_{1}(K)$ extend to $W^{\prime}$. Hence no function in $A_{1}(K)$ assumes a larger value on $W$ than on $K$. It follows that $\delta$ is indeed a continuous homomorphism of $A_{1}(K)$.

We have shown that each compact set $K$ whose interior contains $X$ and is dense in $K$, and all of whose components meet $X$, can be modified slightly to produce another such compact set $K^{\prime}$ with the property that $i_{K^{\prime}}^{*}: M_{A_{1}\left(K^{\prime}\right) *} \rightarrow M_{A\left(K^{\prime}\right) *}$ is onto. Since the collection of such sets $K^{\prime}$ forms a fundamental system of neighborhoods of $X$, it follows from a passage to the projective limit that $i^{*}: M_{A_{0}} \rightarrow M_{A}$ is onto.

Now let $Y$ and $Y^{\prime}$ be distinct components of $M_{A_{1}}$. Considered as a map on $M_{A_{0}}, \hat{Z} \mid Y$ is one-to-one and $\hat{Z}=\hat{Z} \circ i^{*}$ so that $i^{*}$ is certainly one-to-one. If there are infinitely many pairs $(\mu, \nu)$ in $Y \times Y^{\prime}$ such that $i^{*}(\mu)=i^{*}(\nu)$ then some point $(\lambda, \xi)$ is a limit point of such pairs. We may choose a compact set $K$ with smooth boundary, whose interior contains $X$ and is dense in $K$, and such that $i_{K}^{*}(Y)$ and $i_{K}^{*}\left(Y^{\prime}\right)$ belong to different components of $M_{A(K)^{*}}$; say $T$ and $T^{\prime}$ respectively. If $f$ is in $A(K)$, then $\hat{f}$ is analytic on $T-\left(T \cap S_{A(K)^{*}}\right)$ and $T^{\prime}-\left(T^{\prime} \cap S_{A(K)^{*}}\right)$, and the derivative of $\hat{f}$ may be obtained, as in the first part of the proof, by differentiating with respect to the local coordinate $\hat{Z}$. Then the functions $\hat{f} \circ(\hat{Z} \mid T)^{-1}$ and $\hat{f} \circ\left(\hat{Z} \mid T^{\prime}\right)^{-1}$ are analytic in a neighborhood of $\hat{Z}(\xi)=\hat{Z}(\lambda)$ and agree to infinite order there. Now it follows that $\widehat{g}\left(i_{K}^{*}(\xi)\right)=\widehat{g}\left(i_{K}^{*}(\lambda)\right)$ for each $g$ in $A_{1}(K)$, since $A_{1}(K)$ is generated by functions in $A(K)$ and their derivatives. It follows that $i_{K}^{*}(\lambda)=i_{K}^{*}(\xi)$ which is a contradiction. It follows that only finitely many pairs in $Y \times Y^{\prime}$ are not separated by $i^{*}$, as desired.

Finally, suppose that $i^{*}$ is a homeomorphism, and let $f$ be in $A_{0}(U)$ for some open set $U$ containing $X$. As in the proof of Theorem 1 , we may choose a compact set containing $X$ in its interior such that $f \mid(K \cap \hat{Z}(L))$ belongs to $O(\hat{Z}(L)) \mid(K \cap \hat{Z}(L))$ for each component $L$ of $M_{A(K)^{*}}$. As before, we may then conclude that $f$ belongs to $A(K)^{*}$ 
and hence that $f \mid X$ belongs to $A$. Since $U$ is arbitrary, this completes the proof.

Corollary 1. Let $X$ be compact and connected. If $A$ is a complete subalgebra of $\mathcal{O}(X)$ containing the polynomials, then there is a compact connected set $X^{\prime}$ containing $X$ and such that $X^{\prime}-X$ is open and $A=\mathscr{O}\left(X^{\prime}\right)$.

Proof. Let $A_{0}$ be the stable algebra generated by $A$. The Silov idempotent theorem implies that $M_{A}$ is connected and the corollary now follows quickly from Theorems 1 and 2.

The above results have easy applications to questions of approximation on open sets as well. We mention one result that seems particularly striking.

CoROLlary 2. Let $U$ be a connected open set and let $g$ be an analytic function on $U$ that admits no analytic extension to the union of $U$ with any of the bounded components of $C-U$. Then every analytic function on $U$ is the limit, uniformly on compact subsets of $U$, of polynomials in $g$ and $Z$.

\section{REFERENCES}

1. E. Bishop, Subalgebras of functions on a Riemann surface, Pacific J. Math., 8 (1958), 29-50.

2. - Analyticity in certain Banach algebras, Trans. Amer. Math. Soc., 102 (1962), 507-544.

3. - Holomorphic completions, alalytic continuations, and the interpolation of semi-norms, Ann. Math., 78 (1963), 468-500.

4. J.-E. Bjork, Analytic structures, Papers from the Summer Gatherning on Function Algebras at Aarhus, July (1969), 19-28.

5. - On analytic structures in the maximal ideal space of a function algebra, Papers from the Summer Gathering on Function Algebras at Aarhus, July (1969), 29-35.

6. T. W. Gamelin, Uniform Algebras, Prentice-Hall, 1969.

7. - Polynomial approximation on thin sets, Papers from the Symposium on Several Complex Variables, Park City, Utah, (1970), 50-78.

8. H. Royden, Algebras of bounded analytic functions on Riemann surfaces, Acta Math., 144 (1965), 113-142.

9. J. Wermer, Function rings and Riemann surfaces, Ann. Math., 67 (1958), 45-71.

10. - Rings of analytic functions, Ann. Math., 67 (1958), 497-516.

11. W. R. Zame, Algebras of analytic germs, to appear.

Received May 21, 1971.

RICE UNIVERSITY 



\section{PACIFIC JOURNAL OF MATHEMATICS}

\section{EDITORS}

\section{H. SAMELSON}

Stanford University

Stanford, California 94305

\section{R. HOBBY}

University of Washington

Seattle, Washington 98105

\section{J. DugundjI}

Department of Mathematics University of Southern California Los Angeles, California 90007

RICHARD ARENS

University of California

Los Angeles, California 90024

\section{ASSOCIATE EDITORS}
E. F. BECKENBACH
B. H. NeumanN
F. WOLF
K. YOSHIDA

\section{SUPPORTING INSTITUTIONS}

\author{
UNIVERSITY OF BRITISH COLUMBIA \\ CALIFORNIA INSTITUTE OF TECHNOLOGY \\ UNIVERSITY OF CALIFORNIA \\ MONTANA STATE UNIVERSITY \\ UNIVERSITY OF NEVADA \\ NEW MEXICO STATE UNIVERSITY \\ OREGON STATE UNIVERSITY \\ UNIVERSITY OF OREGON \\ OSAKA UNIVERSITY
}

\author{
UNIVERSITY OF SOUTHERN CALIFORNIA \\ STANFORD UNIVERSITY \\ UNIVERSITY OF TOKYO \\ UNIVERSITY OF UTAH \\ WASHINGTON STATE UNIVERSITY \\ UNIVERSITY OF WASHINGTON \\ AMERICAN MATHEMATICAL SOCIETY \\ NAVAL WEAPONS CENTER
}

The Supporting Institutions listed above contribute to the cost of publication of this Journal, but they are not owners or publishers and have no responsibility for its content or policies.

Mathematical papers intended for publication in the Pacific Journal of Mathematics should be in typed form or offset-reproduced, (not dittoed), double spaced with large margins. Underline Greek letters in red, German in green, and script in blue. The first paragraph or two must be capable of being used separately as a synopsis of the entire paper. The editorial "we" must not be used in the synopsis, and items of the bibliography should not be cited there unless absolutely necessary, in which case they must be identified by author and Journal, rather than by item number. Manuscripts, in duplicate if possible, may be sent to any one of the four editors. Please classify according to the scheme of Math. Rev. Index to Vol. 39. All other communications to the editors should be addressed to the managing editor, Richard Arens, University of California, Los Angeles, California, 90024.

50 reprints are provided free for each article; additional copies may be obtained at cost in multiples of 50 .

The Pacific Journal of Mathematics is published monthly. Effective with Volume 16 the price per volume (3 numbers) is $\$ 8.00$; single issues, $\$ 3.00$. Special price for current issues to individual faculty members of supporting institutions and to individual members of the American Mathematical Society: $\$ 4.00$ per volume; single issues $\$ 1.50$. Back numbers are available.

Subscriptions, orders for back numbers, and changes of address should be sent to Pacific Journal of Mathematics, 103 Highland Boulevard, Berkeley, California, 94708.

PUBLISHED BY PACIFIC JOURNAL OF MATHEMATICS, A NON-PROFIT CORPORATION

Printed at Kokusai Bunken Insatsusha (International Academic Printing Co., Ltd.), 270, 3-chome Totsuka-cho, Shinjuku-ku, Tokyo 160, Japan. 


\section{Pacific Journal of Mathematics}

\section{Vol. 42, No. $3 \quad$ March, 1972}

Catherine Bandle, Extensions of an inequality by Pólya and Schiffer for vibrating membranes ................................ 543

S. J. Bernau, Topologies on structure spaces of lattice groups.......... 557

Woodrow Wilson Bledsoe and Charles Edward Wilks, On Borel product measures .......................................

Eggert Briem and Murali Rao, Normpreserving extensions in subspaces of

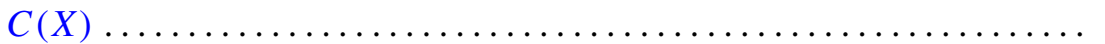

Alan Seymour Cover, Generalized continuation.................. 589

Larry Jean Cummings, Transformations of symmetric tensors .......... 603

Peter Michael Curran, Cohomology of finitely presented groups .......... 615

James B. Derr and N. P. Mukherjee, Generalized quasicenter and

hyperquasicenter of a finite group ...................... 621

Erik Maurice Ellentuck, Universal cosimple isols .................. 629

Benny Dan Evans, Boundary respecting maps of 3-mainfolds .......... 639

David F. Fraser, A probabilistic method for the rate of convergence to the

Dirichlet problem .................................. 657

Raymond Taylor Hoobler, Cohomology in the finite topology and Brauer

groups ..................................... 667

Louis Roberts Hunt, Locally holomorphic sets and the Levi form ........ 681

B. T. Y. Kwee, On absolute de la Vallée Poussin summability............ 689

Gérard Lallement, On nilpotency and residual finiteness in semigroups .... 693

George Edward Lang, Evaluation subgroups of factor spaces........... 701

Andy R. Magid, A separably closed ring with nonzero torsion pic ....... 711

Billy E. Rhoades, Commutants of some Hausdorff matrices ............. 715

Maxwell Alexander Rosenlicht, Canonical forms for local derivations . . . . 721

Cedric Felix Schubert, On a conjecture of L. B. Page ................ 733

Reinhard Schultz, Composition constructions on diffeomorphisms of $S^{p} \times S^{q}$

J. P. Singhal and H. M. (Hari Mohan) Srivastava, A class of bilateral generating functions for certain classical polynomials ....

Richard Alan Slocum, Using brick partitionings to establish conditions which insure that a Peano continuum is a 2-cell, a 2-sphere or an annulus...

James F. Smith, The p-classes of an $H^{*}$-algebra ...

Jack Williamson, Meromorphic functions with negative zeros and positive

poles and a theorem of Teichmuller ................. 